\title{
Oxygen ozone therapy in the treatment of chronic obstructive pulmonary disease: An integrative approach
}

\author{
Emma Borrelli ${ }^{1, *}$, Velio Bocci ${ }^{2}$ \\ ${ }^{1}$ Department of Medical Biotechnologies, University of Siena, Italy \\ ${ }^{2}$ Department of Biotechnologies, University of Siena, Italy
}

Email address:

Ebitaly2007@libero.it (E. Borrelli), bocci@unisi.it (V. Bocci)

\section{To site this article:}

Emma Borrelli, Velio Bocci. Oxygen Ozone Therapy in the Treatment of Chronic Obstructive Pulmonary Disease: An Integrative Approach. American Journal of Clinical and Experimental Medicine. Vol. 2, No. 2, 2014, pp. 9-13.

doi: 10.11648/j.ajcem.20140202.11

\begin{abstract}
A pilot study has been performed on fifty COPD patients: besides using effective drugs, half of the patients have been treated also with major ozonated autohaemotherapy. This treatment has been evaluated during the last two decades and is absolutely atoxic: the treated patients have shown a significant improvement of the six minute walking test and Saint George Respiratory Questionnaire total score. Surprisingly their quality of life was also much improved. Orthodox medications appears to be greatly potentiated when integrated by the autologous infusion of ozonated blood. This combination deserves to be evaluated in an ample clinical trial.
\end{abstract}

Keywords: Ozonetherapy, Chronic Obstructive Pulmonary Disease, Oxygen Radicals

\section{Introduction}

Chronic obstructive pulmonary disease (COPD) is a multi component and heterogeneous disease, with patients differing in terms of clinical presentation and rate of disease progression. Although mainly categorized by airflow limitation that is progressive and not fully reversible, latest severity categorization also includes exacerbation frequency and symptom burden as key features. Moreover, in many patients the disease seems to be associated with several extra-pulmonary manifestations.[1,2]. What is unclear at present is whether these manifestations are directly related to COPD or are just an independent consequence of the exposure to common causal effects such as tobacco smoking and inactivity. The most widely recognized manifestations include the presence of concomitant cardiovascular disease, skeletal muscle dysfunction, osteoporosis, clinical depression and anxiety[3-5]. A major goal in the management of this disease is to ensure that its burden is minimized, such that patients have the best possible health-related quality of life [6]. An ample range and type of drugs such as salmeterol/fluticasone, tiotropium, theophilline, beclometasone, formoterol etc have been tried

with useful but variable results. Recurrent pulmonary infections are treated with antibiotics. However it is now clear that COPD is fundamentally a disease inducing a chronic inflammation with a remarkable increase of reactive oxygen species (ROS), of circulating pro-inflammatory cytokines (IL-1 $\beta$, IL-6,IL-8,IL-18 and $\mathrm{TNF} \alpha$ ) as well as acute-phase proteins such as $\mathrm{C}$ reactive protein (CRP) and serum amyloid.

Therefore it is clear that COPD is a very complex disease where the chronic inflammation of the lungs [7,8] extends to the whole body with deranged metabolic cardiovascular and bone diseases. Beside smoking cessation, which is a must, an anti-inflammatory treatment associated to a suppression of pulmonary inflammation appears essential to slow down the progression of the disease [8]. During the last two decades the biological and molecular mechanism of action of oxygen ozone therapy have been fully clarified [9 -12] and its rationale is clarified in the next session. It has been emphasized what this treatment has unique properties in reducing inflammation and upregulating the cellular antioxidant system. Consequently the optimal anti COPD medical therapy integrated by ozonetherapy is suggested here as novel approach.

The rationale of ozone therapy

The chronic oxidative stress can be corrected by using ozone-therapy

Among the numerous complementary methods, only one is specifically able to correct the chronic oxidative stress. 
Among the numerous complementary methods, only one is specifically able to correct the chronic oxidative stress. Preclinical and clinical studies performed with ozone-therapy have been performed in many countries and in Germany more than one million sessions are successfully held every year without any side effects.

Ozone, like other gases as $\mathrm{NO}, \mathrm{CO}, \mathrm{H}_{2} \mathrm{~S}$ and $\mathrm{H}_{2}$, although intrinsically toxic, when properly used in well determined small concentrations, has become important medical gas [13-14]. Usually $100-150 \mathrm{ml}$ of blood of the patient, anticoagulated with $\mathrm{Na}$ Citrate, are collected in a sterile glass ozone-resistant bottle and an equal volume of a gas mixture $\left(96 \% \mathrm{O}_{2}-4 \% \mathrm{O}_{3}\right)$ is added at a precisely determined ozone concentration within $0.21-0.84 \mathrm{mM}$ per $\mathrm{ml}$ of blood .The ozonated blood is then infused into the donor patients within 2-3 min. The initial dose is minimal and, for avoiding any side effects, is slowly upgraded up to 0.84 $\mathrm{mM}$ throughout the necessary 30-40 sessions, twice weekly. The axiom:"start low, go slow" proves to be ideal and under these conditions, ozone behaves as an acceptable stressor. Indeed ozone therapy induces a series of graduated small oxidative stress acting on all organs and able to reactivate the potent defense system, which counteracts the deleterious chronic oxidative stress induced by COPD .

The high reactivity and solubility of ozone in the water of plasma allows its exhaustion in one min while it generates two crucial messengers such as $\mathrm{H}_{2} \mathrm{O}_{2}$ and 4-hydroxynonenal (4-HNE) finally responsible for eliciting the well defined biochemical and molecular reactions responsible for the biological activities. 4-HNE readily forms an adduct with the Cys34 of albumin or with glutathione and this compound is consequently be able to reach most of cells of the body and to reactivate the antioxidant defenses.

The real molecular mechanisms of antioxidant activation

Alkenal adducts are able to react with the Kelch-like ECH-associated protein 1 (Keap 1)-NF-E2-related factor 2 (Nrf2) system that is present in the cell cytosol with the role of antagonizing oxidative and electrophilic insults. In detail, keap1 is a protein molecule with many - SH groups that are important for the repression of Nrf2 activity. Normally, the complex Nrf2-Keap1 has a half life of about 20 minutes because keap 1 is readlily ubiquitinated and digested in the proteasome. However, the alkenal interaction with both Cys 272 and 288 of Keap-1 allows the release of $\mathrm{Nrf2}$, which escapes proteosomal degradation and traslocates into the nucleus, heterodimerizes with a small Maf protein and binds to the antioxidant Response Element (ARE or EHRE) on DNA [15].

On this basis, it is clear that Nrf2 is now correctly recognized as the key cellular defense system against oxidative and xenobiotic stresses. Such a crucial event is able to upregulate the synthesis of relevant enzymes.

The reactivation of innate defense system leads to the synthesis of a number of antioxidant enzymes able to counteract the chronic oxidative stress:

a) activation of the gamma-glutamyl-cysteine ligase and of GSH synthase allows a marked increase of the GSH intracellular level. The enhanced GSH/GSSG ratio allows an important protection against ROS ;

b) activation of the synthesis of antioxidant enzymes able to detoxify an excess of ROS such as catalase, SOD,GSH-peroxidases, GSH-reductase,NADPH-quinoneox idoreductase(NQO1), cytochrome P450 monooxygenase system and HSP70 ;

c) the upregulation of Heme-oxygenase $1(\mathrm{HO}-1)$ is also very protective and the trace of $\mathrm{CO}$ in combination with NO allows vasodilatation of ischemic tissues;

d) the enhancement of the synthesis and levels of phase II enzymes such as GSH S-transferases, UDP-glucuronosyltransferases, N-acetyltransferases ans sulfotransferases ;

e) inhibition of cytokine mediated inflammation via the induction of leukotriene B4 reductase ;

f) reducing iron overload and subsequent oxidative stress induced via elevated ferritin and bilirubin as a lipophilic antioxidant ;

g) the repetition of graduated small oxidative stresses induces a multiform adaptative response. Moreover, during the ozone therapy sessions there is an increased release of adrenocorticothropic hormone, followed by cortisol from the cortex of the adrenal glands as a consequence of the liberation of corticotrophin releasing factor from the hypothalamus. Such a response is responsible for an improved feeling of well being reported by the majority of patients.

The efficacy of the mentioned orthodox drugs associated with the safe and valid support of the ozonated autohaemotherapy proves that the concept of integrated medicine is the best combination because it correctly associates suitable drugs with the critical stimulus of reactivating the natural defenses.

\section{The absolute lack of toxicity of ozone-therapy}

Gaseous ozone can be harmful at even low concentrations of part per million, affecting especially the eyes and respiratory systems. Administration of ozone by aerosol is toxic and must be avoided, as well as the intravenous administration of the oxygen-ozone gas mixture. On the contrary, very small and precisely determined ozone dosages during ozone therapy do not procure any acute or late side effects. In fact addition of ozone to blood happens ex vivo and the minimal amount of ozone acts as a pro drug and within 2-3 min is exhausted in small parts by the plasma antioxidants by generation of $\mathrm{H}_{2} \mathrm{O}_{2}$ and alkenals .

The performance of ozonetherapy twice weekly for at least five months ( about 40 sessions) also allows to improve the vascular system and to enhance the oxygenation of ischemic tissues. Moreover, such a bio oxidative procedure, can be continued with a weekly session for life with an excellent compliance of patients . On this basis it was decided to start a preliminary clinical investigation on COPD patients. 


\section{Subjects and Methods}

In this study we enrolled 50 patients affected by moderate/ severe COPD, which was diagnosed according to the current guidelines [16]. All of them were ex smokers and referred a score 2-3 according to MRC classification. They were observed in a stable phase of the disease, free from exacerbations in the 4 weeks before starting the study protocol and under regular pharmacologic therapy ( inhaled long acting beta2 agonists/ corticosteroids LABA/ICS and/or tiotropium in all patients).

Before starting the study each patient underwent a clinical assessment, chest X-ray, detailed pulmonary function test (PFT), resting arterial blood gas analysis. Exclusion criteria were unstable ischemia and uncontrolled pathology.

Patients were enrolled in two groups: group A ( 25 patients) underwent a cycle of oxygen ozonetherapy ( major ozonated autohaemotherapy, $\mathrm{O}_{3}$-AHT) twice a week for the first 5 weeks, thereafter a single treatment every week for other 10 weeks. Group B ( 25 patients) served as control and not received treatments otherwise the standard therapy with inhaled beta2 long acting bronchodilators and/or corticosteroids .In all patients we measured before and after the end of the study: 1) Pulmonary FunctionTest 2 )Resting Arterial blood gas 3) exercise tolerance by 6 min walking test (6MWT) 4) dyspnea index by Borg dyspnea scale 5) health status, evaluate by the St. George Respiratory questionnaire ( SGRQ). The patient's characteristics at the start of the study are reported in table 1 .

Table 1. Baseline characteristics (mean $\pm S D$ ) of the patients enrolled in Group A (ozone treatments) and Group B (control group).

\begin{tabular}{ccc}
\hline & GROUPA & GROUP B \\
\hline Number & 25 & 25 \\
Gender (M/F) & $15 / 10$ & $17 / 8$ \\
BMI & $26.8 \pm 3.1$ & $26.2 \pm 2.7$ \\
FEV1 $\%$ pred $)$ & $51.1 \pm 18$ & $50.7 \pm 17.4$ \\
FVC $(\%$ pred $)$ & $76.2 \pm 16.4$ & $78 \pm 15.2$ \\
RV(\% pred) & $130 \pm 37.1$ & $132 \pm 40$ \\
TLC $(\%$ pred $)$ & $106.6 \pm 20.3$ & $107.1 \pm 21$ \\
COPD severity $*$ & & \\
Moderate & 15 & 17 \\
Severe & 10 & 8 \\
\hline
\end{tabular}

FEV1 = forced expiratory volume in one second; $F V C=$ forced vital capacity; RV= residual volume $; \mathrm{TLC}=$ total lung content; $*$ according to GOLD classification. All comparison between group was not statistically significant.

An informed consent for the oxygen ozone therapy was obtained in patients of group A. The study was approved by the Siena University Hospital Ethical Committee, and it was conducted in accordance to the ethical consideration of the World Medical Association ( Helsinki Declaration).

Oxygen ozonetherapy protocol

Oxygen ozonetherapy procedure O3-AHT was carried out as follows: $225 \mathrm{~mL}$ of blood were drawn by vacuum from an antecubital vein into a sterile glass bottle (Ozonosan, Iffezeim, Germany) in which $25 \mathrm{~mL}$ of $3.8 \% \mathrm{Na}$ citrate solution (Galenica Senese Industries, Siena, Italy) as an anticoagulant, had been previously added so that the blood/citrate volume ratio was 9:1. After blood withdrawal, the bottle was momentarily disconnected leaving the venous access open by a saline infusion. The use of $225 \mathrm{~mL}$ of blood was unrelated to the subject's body mass, rather, it was related to the dROM values in plasma and to previously reported protocols [12]. A corresponding volume $(225 \mathrm{~mL})$ of gas was immediately added with an $\mathrm{O}_{3}$ concentration of $20 \mathrm{micrograms} / \mathrm{ml}$ for the first four treatments, thereafter the concentration of ozone was 40 micrograms $/ \mathrm{mL}$ of gas for the other 16 treatments. Ozone was produced by an Ozonline $80 \mathrm{E}$ generator (Medica srl, Bologna, Italy), in which $\mathrm{O}_{3}$ concentration was measured photometrically in real time and checked by iodometric titration according to the rules established by the International Ozone Association.

The gas was immediately and continuously mixed with the blood in the bottle for at least 5 minutes and with gentle rotating movement to avoid foaming. Due to the blood viscosity, the gas mixture does not instantaneously come into contact with the whole blood mass, thus this mixing time is necessary. Indeed, the $\mathrm{pO}_{2}$ value only after this mixing period usually reaches a plateau level (of about $500 \mathrm{mmHg}$ ). During these 5 minutes of mixing the ozone totally reacted with both the potent antioxidants of plasma and the unsaturated lipids bound to albumin, generating a small amount of hydrogen peroxide and alkenals. These two messengers were responsible for eliciting crucial biochemical reactions on both erythrocytes and within cells when the hyper-oxygenated ozonated blood was re-infused into the patient. At this point, the hyper-oxygenated ozonated blood was re-infused by promptly substituting the saline infusion with it. Reinfusion was accomplished in about 15-20 minutes and the whole procedure was carried out in approximately 40 minutes. $\mathrm{O}_{3}$-AHT was carried out in an out-patient setting twice weekly (on Tuesday and on Friday) for the first 5 weeks, thereafter a single treatment a week for other 10 weeks.

\section{Statistical Analysis}

Statistical analyses were performed using statistical package SPSS and the Student $t$ test for the intragroup ( before and after the end of study) and intergroup ( before study, group A vs group B and after the end of study, group A vs group B) comparison of parameters. $\mathrm{P}<0.01$ was considered significant.

\section{Results}

In the group A there was a significant increase in 6MWT ( $417.5+/-107.4 \mathrm{~m}$ before the study vs $493.8+/-106 \mathrm{~m}$ at the end of study, $p<0.01$ ) and a significant decrease in the degree of dyspnea measured by Borg scale after the 
6MWT ( $4.1+/-2.4$ vs $3+/-2.1, \mathrm{p}<0.01)$ and in SGRQ activity impact and total scores ( activity: $65.4+/-16.3 \%$ vs $54.2+/-15 \%$ p $<0.01$; impact: $38.4+/-18.2 \%$ vs $30.1+/-$ $16.4 \%, \mathrm{p}<0.01$; total score: $48.9+/-16.0 \%$ vs $32.2+/-$ $12.1 \%, \mathrm{p}<0.01$ ) (Table 2). No statistical difference were observed in the pulmonary function test and resting arterial blood gas before and after ozone therapy.

Table 2. Modification of parameters in Group A and Group B before and after the study.

\begin{tabular}{llll}
\hline & Group A & Group B & p \\
\hline 6MWT before (m) & $417.5 \pm 107.3$ & $411.4 \pm 109.2$ & $\mathrm{~ns}$ \\
6MWT after (m) & $493.8 \pm 105.4$ & $407.3 \pm 104.4$ & $\mathrm{P}<0.01 \mathrm{a}, \mathrm{b}$ \\
BORG SCALE before & $4.1 \pm 2.4$ & $4.3 \pm 2.1$ & $\mathrm{~ns}$ \\
BORG SCALE After & $3 \pm 1.2$ & $4.3 \pm 2.1$ & $\mathrm{P}<0.01 \mathrm{a}$ \\
SGRQ & & & \\
Activity before (\%) & $65.4 \pm 16.3$ & $66.1 \pm 17.4$ & $\mathrm{~ns}$ \\
Activity after (\%) & $54.2 \pm 15$ & $62.4 \pm 16.6$ & $\mathrm{P}<0.01 \mathrm{a}$ \\
Impact before (\%) & $38.4 \pm 18.2$ & $37.6 \pm 17.1$ & $\mathrm{~ns}$ \\
Impact after (\%) & $30.1 \pm 16.4$ & $34.5 \pm 17.9$ & $\mathrm{P}<0.01 \mathrm{a}$ \\
Total score before(\%) & $48.9 \pm 16$ & $47.3 \pm 16.4$ & $\mathrm{~ns}$ \\
Total score after(\%) & $32.2 \pm 12.1$ & $49.9 \pm 14.3$ & $\mathrm{P}<0.01 \mathrm{a}, \mathrm{b}$ \\
\hline
\end{tabular}

$6 \mathrm{MWT}=$ six minutes walking test; Borg SCALE= Borg dyspnea scale;SGRQ= Saint George Respiratory Questionnaire (items activity, impact,total score);before=value before study;after= value at the end of the study; $\mathrm{a}=$ intragroup comparison before versus after study, group $\mathrm{A}$; $\mathrm{b}=$ intergroup comparison Group A versus Group B, after study.

In the control group, no significant difference were observed before and after the study in all parameters recorded. The intergroup comparison in 6MWT and SGRQ total score at the end of study showed a significant difference (6MWT at the end of study: group A $493.8+$ $106 \mathrm{~m}$ vs group B $407.3+/-104.4 \mathrm{~m}, \mathrm{p}<0.01$; SGRQ total score at the end of study :group A $32.2 \pm 12.1$ vs group B $49.9 \pm 14.3, \mathrm{p}<0.01)$.

\section{Side Effects and compliance}

We have observed only temporary face redness in a small percentage of patients (3\%) during the treatment with oxygen ozone therapy. Patients reported an improvement of their general conditions, particularly in terms of increased efficiency, mental concentration and memory.

\section{Discussion}

Traditionally, the severity of COPD was equated with air flow limitation, as measured by impairment in forced expiratory volume in one second (FEV1) and treatment and management of the disease was also largely based on spirometric assessment. However, because COPD is a multicomponent disorder, structural and functional changes take place in other organs, as well as in the lungs [17].Therefore, airflow limitation alone does not reflects the full burden of COPD such as dyspnea, exercise intolerance and impairment of health related quality of life [18].Oxygen ozone therapy represents a systemic therapy with a deep impact with many organs, and it is not surprising that it was a significant improvement in patient's quality of life and exercise tolerance.

Three years ago we performed the ozonated autohaemotherapy in two COPD patients also affected by age-related macular degeneration, who showed an unexpected improvement. Consequently, it was decided to more accurately examine the outcome in this pilot trial. The patients themselves were enthusiastic about their improved quality of life. It appears evident how the most suitable medical treatment integrated by ozonated autohaemotherapy was responsible for the improvement. Now one autohaemotherapy per week is continued with the satisfaction of patients. It must be clarified that the minimal dosage of ozone $(20 \mu \mathrm{g} / \mathrm{mL}$ or $0.42 \mu \mathrm{mol} / \mathrm{mL}$ gas $)$ acts as a pro-drug because, in less than five minutes, is totally exhausted during mixing ex vivo in the glass bottle. Its relevant messengers are $\mathrm{H}_{2} \mathrm{O}_{2}$, which acts biochemically mostly on the erythrocytes shifting the oxyhaemoglobin curve to the right, plus an improved NO production by the endothelium. Even more relevant is the effect caused by submicromolar levels of 4-HNE with hydroperoxides that, after infusion of the ozonated blood, act as a well tolerated oxidative stress on a variety of body cells reactivating both the defence system consisting of the up regulation of antioxidant system plus phase- 2 enzymes. The progressive improvement of the redox homeostasis appears the critical factors justifying the improvement of the quality of life. Indeed the patients refer to feel much better after receiving the first 7-12 infusions, that is from 1575 to $2700 \mathrm{ml}$ of ozonated blood. Naturally ozone as a gas is neither inhaled, nor directly infused because ozone is so reactive to be partly neutralized by plasma antioxidants and partly consumed during the peroxidation reaction of polyunsaturated lipids occurring ex vivo. This must be said because by 2006 the dogma that ozone is always toxic has been fully refuted. Consequently it appears useful to inform that the ozonetherapeutic integration can represent a novel approach for the COPD patients: it would be most important to verify our preliminary data in an ample clinical trial.

\section{References}

[1] Global Initiative for Obstructive Lung Disease. http://www.goldcopd.com. Last Access Sep 21, 2012

[2] Decramer M, Janssens W, Miravitlles M. Chronic obstructive pulmonary disease. Lancet 2012; 379: 1341-51.

[3] Mannino DM,Buist AS.Global burden of COPD: risk factors,prevalence and future trends. Lancet 2007;370:765-73.

[4] Hogg Jc, Timens W. The pathology of chronic obstructive pulmonary disease. Annu Rev Pathol 2009;4:435-59.

[5] Barnes PJ, Celli BR. Systemic manifestations and comorbidities of COPD. Eur Resp J 2009;33: 1165-85. 
[6] Sinden NJ, Stockley RA. Systemic inflammation and comorbidity in COPD: a results of "overspill" of inflammatory mediators from the lungs? Review of the evidence. Thorax 2010;65:930-936.

[7] Barnes PJ, Shapiro SD, Pauwels RA. Chronic obstructive pulmonary disease: molecular and cellular mechanisms. Eur Resp J 2004;22:672-688

[8] Thomsen M, Ingebrigtsen TS, Marott JL, Dahl M, Lange P, Vestbo J, Nordestgaard BG. Inflammatory biomarkers and exacerbations in chronic obstructive pulmonary disease. JAMA $201312 ; 309$ (22) : 2353-61

[9] Bocci V, Borrelli E, Travagli V, Zanardi I. The ozone paradox: ozone is a strong oxidant as well as a medical drug. Medic Res Rev 29: 646-82, 2009

[10] Bocci V. A new method for the activation of the cellular antioxidant system. Oxidants and Antioxidants in Medical Science 20132 (3) 149-154.

[11] Bocci V. Ozone. A new medical drug. 2nd Edition. Springer Editor. 2011, pp. 1-315

[12] Borrelli E, Bocci V. Basic biological and therapeutic effects of ozonetherapy in medicine In: Encyclopedia of Life Support Systems (EOLSS), Developed under the Auspices of the UNESCO, Eolss Publishers, Oxford ,UK, [http://www.eolss.net].

[13] Bocci V. Is it true that ozone is always toxic? The end of the dogma. Toxicol. Appl. Pharmacol. 2006; 216: 493-504.
[14] Bocci V, Zanardi I, Michaeli D, Travagli V. Mechanisms of action and chemical-biological interactions between ozone and body compartments: a critical appraisal of the different administration routes. Current Drug Therapy 2009, 4: 159-175.

[15] Pecorelli A, Bocci V, Acquaviva A, Belmonte G, Gardi C, Virgili F, Ciccoli L, Valacchi G. Nrf2 activation is involved in ozonated human serum upregulation of HO-1 in endothelial cells. Toxicology and Applied Pharmacology 2013; 267:30-40

[16] Qaseem A, Wilt TJ, Weinberger SE, Hanania NA, Criner G, van der Molen T, Marciniuk DD, Denberg T, Schünemann H, Wedzicha W, MacDonald R, Shekelle P; American College of Physicians; American College of Chest Physicians; American Thoracic Society; European Respiratory Society . Diagnosis and management of stable chronic obstructive pulmonary disease: a clinical practice guideline update from the American College of Physicians, American College of Chest Physicians, American Thoracic Society,and European Respiratory Society. Ann Intern Med 2011; 155: 179-91

[17] Wegner R, Jörres RA, Kirsten DK, Magnussen H. Factor analysis of exercise capacity, dyspnoea ratings and lung function in patients with severe COPD. Eur Respir $J$ $1994 ; 7: 725-9$

[18] O'Donnel DE.Hyperinflation, dyspnea and exercise intolerance in chronic obstructive pulmonary disease. Proc Am Thorac Soc 2006; 3: 180-84. 